has no foundation in fact. There seems to be an extraordinary misconception on the part of the profession as to the present status of the operation on the ganglion or root. It is usually represented as a court of last resort, a sort of kill-or-cure method, as a result of which the patient, if he survives, will be paralyzed on one side of the face and will lose his eyesight. Because of the frame of mind in which the patient consults the surgeon, the former needs some reassuring. In stating the risks of the operation, I shall refer only to my own clinic. Since 1901, there have been, all told, four deaths in 160 operations. Two of these were due to apoplexy, and all of them were among. subjects of the 121 intracranial operations. There was one death in 1904, and one in 1910, and there were two in 1912. In a series of eighty-seven consecutive operations there have been no deaths since 1912 .

Over other radical operations, such as the removal of the ganglion, the Abbe operation or the Hutchinson operation, avulsion of the sensory root has so many advantages that it should be and is recognized as the operation of choice. It. is easier of execution than any other, it subjects adjacent structures to no risk, and it is attended with a smaller percentage of corneal complications and with a lower mortality. On the whole, it is the most satisfactory surgical procedure with which I have had to deal.

1724 Spruce Street.

\section{THE CAUSE OF LUMBAR PUNCTURE HEADACHE}

RUSSELL G. MACROBERT, M.B. (ToR.) Associate Physician, Neurological Institute NEW YORK

Lumbar puncture headache differs from all others in that, being present when the patient is sitting up, it completely disappears when he lies down. It is throb-

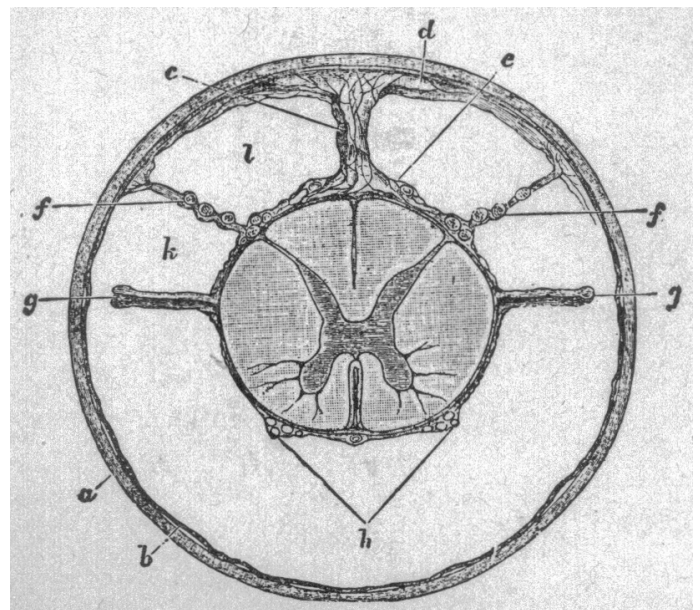

Fig. 1.-Section of the spinal cord within its membranes and subarachnoidal space which contains the cerebrospinal fluid (Key and Retzius, Schäfer's Histology): $a$, dura mater; $b$, arachnoid; $k, l$, sub arachnoid space.

bing and severe, and felt mostly in the frontal and occipital regions. No drugs give sufficient relief to allow its victim to walk about and attend to his affairs, or even to sit up. The pain comes on quickly when he sits up, being fully present in twenty seconds. It takes about the same length of time to subside when he lies down. This situation usually persists for about seven days with full severity, and then ends somewhat abruptly in the course of twenty-four hours.

The only effective treatment is a week's stay in bed with the head low. While in this position the patient is quite comfortable, and there are no clinical signs of trouble present.

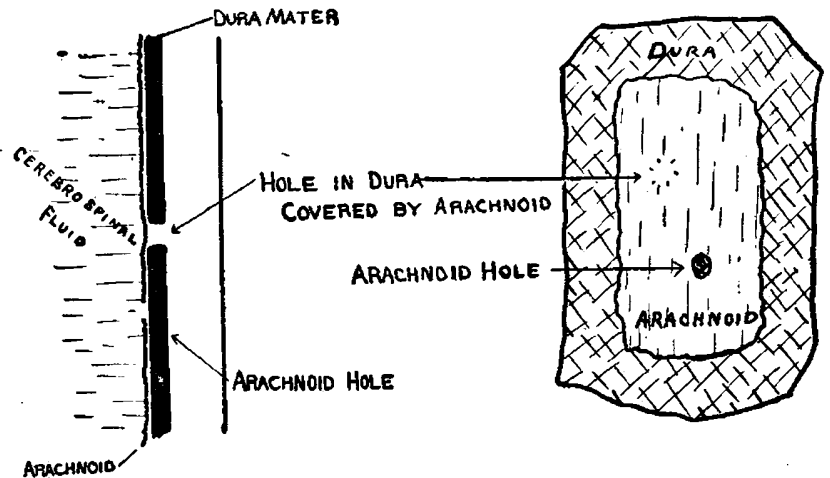

Fig. 2.-Spinal membranes with normal closure of puncture hole: no epidural leakage; no headache.

Regarding causative or influencing factors of the headache, various speculations have been made concerning the importance of such points as the rapidity with which the fluid is withdrawn; the position of the patient during the puncture; the degree of pressure existing in the spinal fluid at the time of puncture; the disease condition of the patient, and his age. All these points are now generally regarded as unimportant.

There are three other points that linger with some observers, and these have been made interesting by discussion. They are: (1) lying flat on the back for twenty-four hours following puncture; (2) performance of puncture when the fluid shows signs of active syphilis, and (3) the amount of fluid withdrawn.

1. It has been our custom at the Neurological Institute to have the patient lie flat on his back for twentyfour hours following lumbar puncture. The small amount of fluid collected for examination must by this time be easily compensated for, and the chances

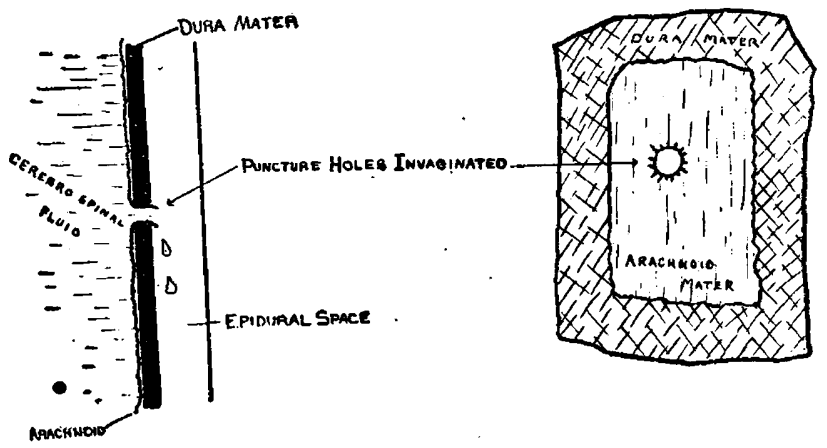

Fig. 3.-Nonclosure of puncture hole, because of arachnoid tissue being pulled through dural opening as needle was withdrawn, resulting in prolonged epidural leakage and lumbar puncture headache.

of headache appearing were expected to be considerably lessened. In the spring of 1915 , by a series of thirty consecutive cases in which this procedure should be strictly adhered to, I determined to establish its value. Twelve of the thirty developed the typical severe headache.

2. Some information concerning these thirty cases is included here, to illustrate the fallacy of a statement 
frequently heard in connection with the second point, regarding the performance of puncture when the fluid shows signs of active syphilis. This statement is to the effect that headache is unlikely to occur in these cases. In the accompanying table only eight spinal

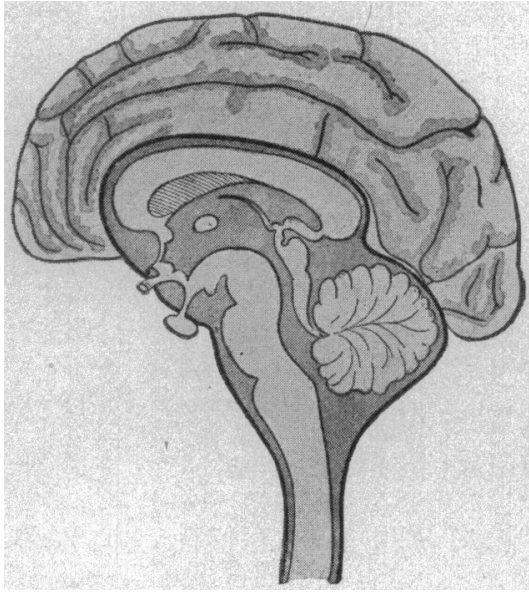

Fig. 4.- Location of the cisternae and and ology). fluids may be considered as showing signs of active syphilis, and yet it was in three of these that headache occurred. If this percentage, 37.5 , is compared with the 40 per cent. in the whole series, it becomes evident that we may not regard this point as an influencing factor of the headache.

3. The third point-- the amount of fluid withdrawn - is interesting. The liability for headache to occur when only 2 c.c. of fluid are withdrawn, as readily as when 20 c.c. are withdrawn, has often been demonstrated. This has led some to assume and contend that headache did not depend on loss of cerebrospinal fluid. To support their contention, they have cited the fact that headache is not a frequent complaint after spinal cord operations, although much fluid is usually lost during these operations. The fallacy of this illustration becomes apparent when we recall that after spinal cord operations, the patient is kept on his back for at least two weeks; lumbar puncture headache is not in evidence while a patient is on his back, and the situation responsible for it, whatever it is, is never present, even in the worst cases, for as long as two weeks.

Whereas we are far from having any proof that loss of fluid does not cause lumbar puncture headache, and the consensus of opinion is that in some way it must be responsible, it seems ridiculous that when probably 60 c.c. or more are secreted in a day, an alteration in the intracranial situation sufficient

to produce violent headache of seven or more days' duration can be made by the removal of from 2 to 5 c.c.

After a consideration of these facts, it was evident that some new point of view must be obtained in order to solve the problem. A critical analysis of the whole puncture phenomenon brought to light a factor that seems not to have been previously considered. It occurred in answer to the question, After the puncture, is everything within as it was before, with the excep-

OCCURRENCE OF HEADACHE IN THIRTY CONSECUTIVE PUNC'IURES

\begin{tabular}{|c|c|c|c|c|c|c|c|}
\hline \multirow{2}{*}{ No. } & \multirow{2}{*}{ Patient } & \multirow{2}{*}{ Diagnosis } & \multicolumn{3}{|c|}{ Sp:nal Fluid } & \multirow{2}{*}{$\begin{array}{l}\text { Head- } \\
\text { ache }\end{array}$} & \multirow{2}{*}{ No. } \\
\hline & & & Wass. & Glob. & Cells & & \\
\hline 1 & B. & Tabes.... & - & $w+$ & 3 & No & \\
\hline 2 & s. & Paresis................ & + & + & 51 & Sevcre & \\
\hline 3 & G. & Cerebrospinal syphilis & + & + & $3 \overline{3}$ & Severe & II \\
\hline 4 & C. & rabes................. & + & + & 35 & No & \\
\hline 5 & h. & Cerebrospinal syphilis & + & + & 20 & No & \\
\hline 6 & D. & Paresis............... & + & + & 144 & No & \\
\hline 7 & $\mathrm{~J}$. & Lateral sclerosis......... & - & - & 1 & No & \\
\hline 8 & H. & Chorea................. & - & $\ldots$ & 1 & Severe & III \\
\hline 9 & G. & Epilepsy............. & - & - & 0 & No & \\
\hline 10 & K. & Cerebrospinal syphilis & -. & - & 0 & No & \\
\hline 11 & C. & Pernicious anemia.... & - & - & 0 & Severe & IV \\
\hline 12 & B. & Multiple sclerosis....... & - & - & 5 & Severe & $\mathrm{V}$ \\
\hline 13 & $\mathbf{R}$. & Cerebrospinal syphilis: & - & - & 1 & Severe & VI \\
\hline 14 & R. & Tabes................. & - & + & 2 & No & \\
\hline 15 & B. & Hysteria............ & - & - & $\overline{0}$ & No & \\
\hline 16 & $\bar{P}$. & Neurasthenia......... & $\rightarrow$ & - & 0 & Severe & VII \\
\hline 17 & G. & Old hemiplegia.... & - & $\ldots$ & 4 & No & \\
\hline 18 & A. & Paresis.......... & + & + & $100^{\circ}$ & Severe & VIII \\
\hline 19 & D. & Paresis........ & + & + & 8 & No & \\
\hline 20 & $\mathbf{K}$. & $?$ & - & - & 0 & No & \\
\hline 21 & R. & Neurasthenia...... & - & - & 1 & No & \\
\hline 22 & c. & Seiatic neuritis........... & - & - & 3 & No & \\
\hline 23 & $\mathrm{R}$. & Cerebrospinal syphilis & - & - & 2 & No & \\
\hline 24 & c. & Tabes................ & - & $w+$ & $\overline{8}$ & Severe & IX \\
\hline 25 & G. & Cerebrospinal syphilis & - & - & 23 & No & \\
\hline 26 & $\mathrm{Z}$. & Wood alcohol......... & - & $\mathbf{w}+$ & 0 & Severe & \\
\hline 27 & $\ddot{H}$. & Fpilepsy............. & -- & - & 0 & No & \\
\hline 28 & $\mathrm{z}$. & Chorea................. & - & - & 1 & Severe & $\mathrm{XI}$ \\
\hline 29 & $\mathrm{P}$. & Cerebrospinal syphilis & - & - & 0 & Severe & XII \\
\hline 30 & R. & Paresis............... & + & + & 44 & No & \\
\hline
\end{tabular}

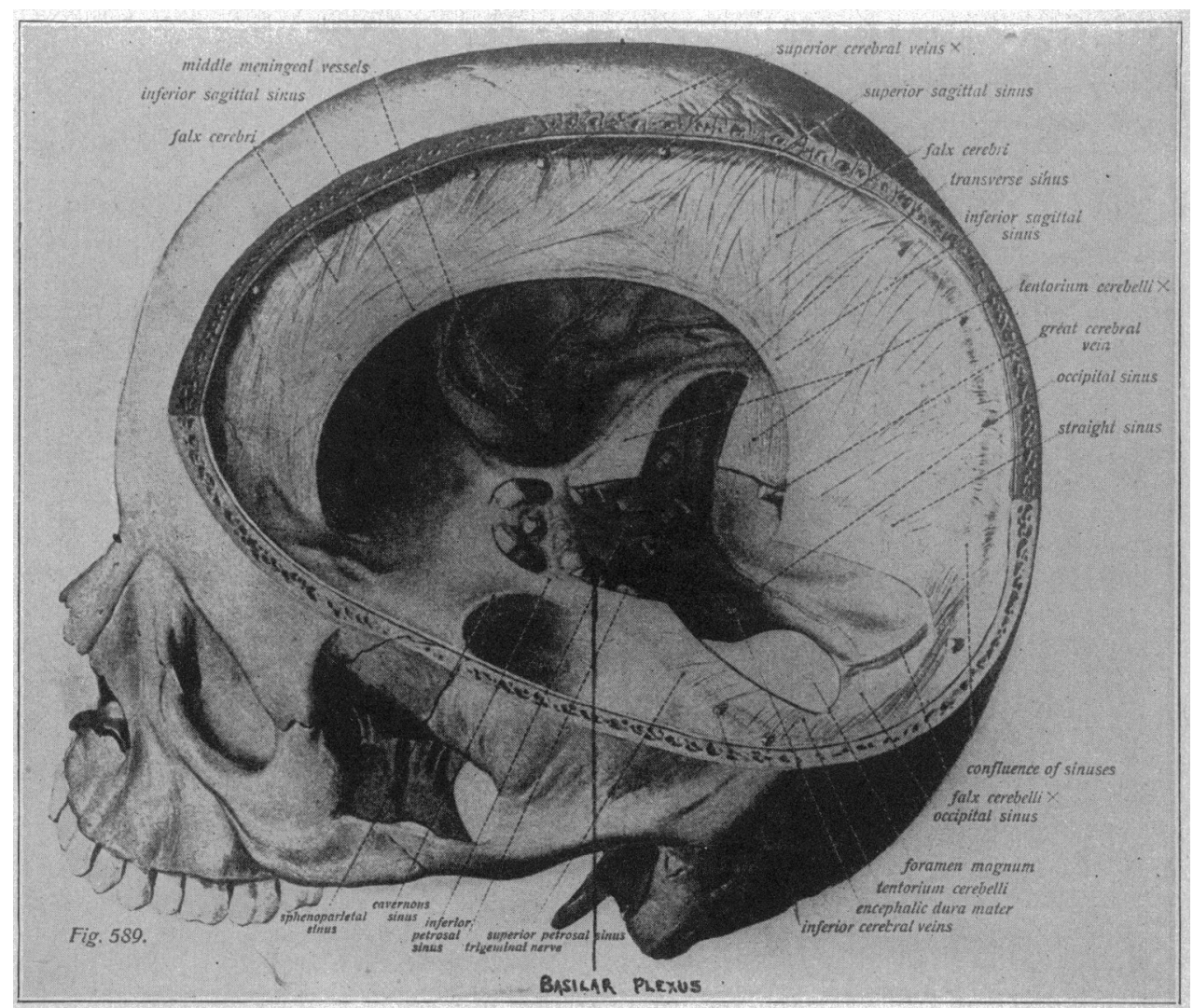

Fig. 5.-Location of basilar plexus of veins (from Sobotta-McMurrich). tion of an absence of a few cubic centimeters of spinal fluid?

To obtain fluid by puncture, the needle must pierce two membranes, the dura and the arachnoid. The dura forms a rigid, tough, fibrous sac, just within the 
vertebral canal. The arachnoid tissue, which is nonvascular and delicate in texture, is full and loose, and it is in close apposition to the dura. The fluid is contained in a space between the arachnoid and the pia mater, the latter membrane closely investing the spinal cord.

We are so accustomed to withdraw blood from a vein without an afterthought as to its closure that I suppose we have assumed the same mental attitude, unconsciously, after lumbar puncture. But in the latter instance we have punctured a fibrous sac distended with fluid - made a hole in a stiff membrane that has no contractile tissue.

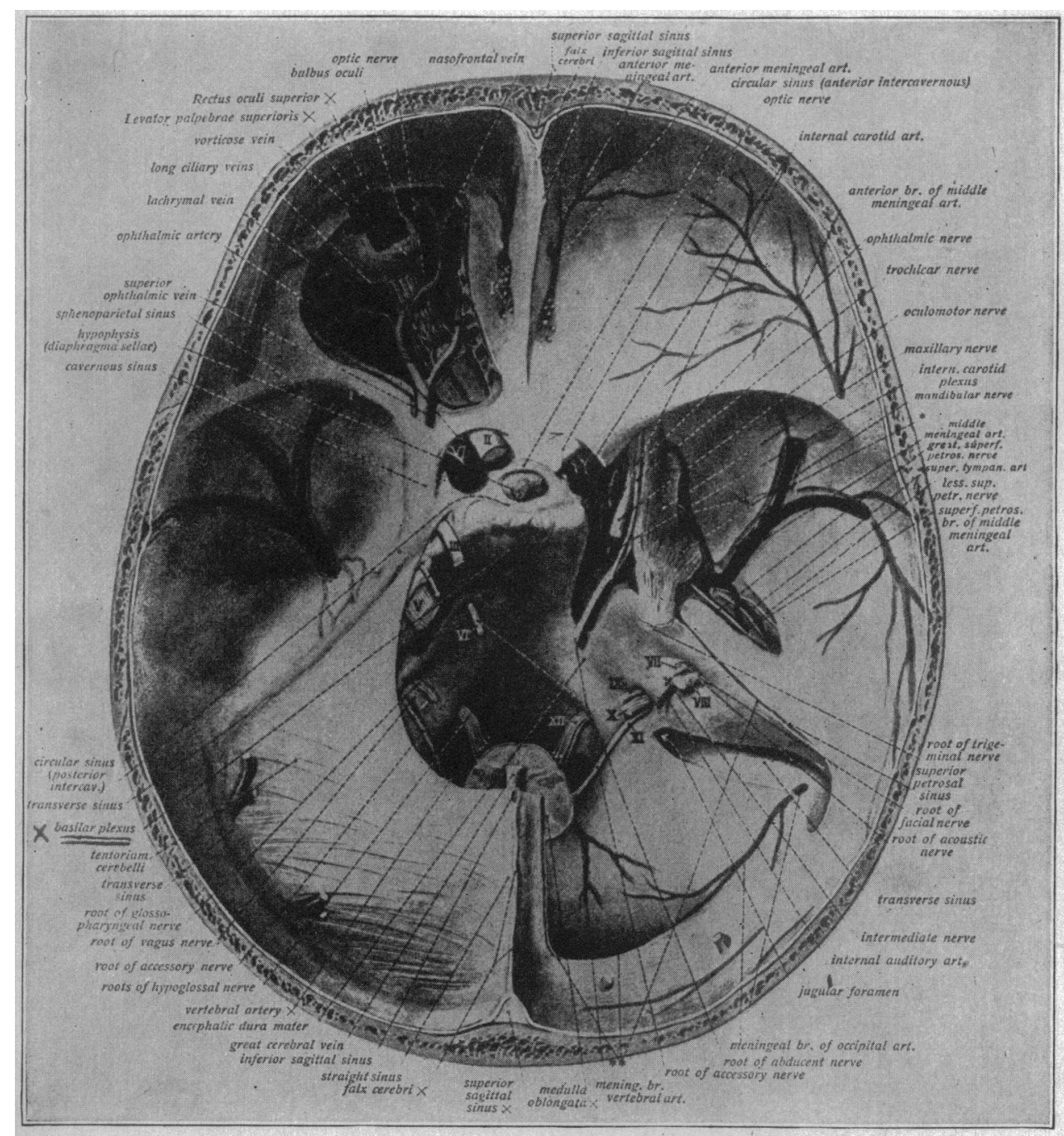

Fig. 6.-Location of basilar plexus on clivus of sphenoid and occipital bones (from Sobotta-McMurrich). mate. a dural opening. by an intact area of the arachnoid membrane, as the puncture holes, being small, are unlikely to approxi-

If the puncture hole is not blocked, it is because the delicate arachnoid tissue clings around the departing needle, and its hole is pulled into and through the hole in the dura. There it impinges, and this invagination forms a spout or wick for the easy drainage of the whole cerebrospinal fluid sac, and also prevents the rapid healing, which would otherwise occur, of so small

All the fluid secreted by the choroidal glands during seven or eight days, the time seemingly necessary for the hole to close by tissue growth, will be lost by leakage into the epidural space, where it can be absorbed readily, because the epidural space of the spinal canal is comparatively very large, and contains only loose connective tissue, with venous plexuses and lymph channels.

It is evident, in the light of the foregoing facts, that the amount of fluid collected in the test tube will be no indication whatever of the great loss that occurs, when a puncture hole does not become properly occluded when the - needle is withdrawn. An instance occurred at the Neurological Institute in January, 1918, which glaringly demonstrates the importance of this occult. leakage into the epidural space, and which also indirectly led me first to consider the possibility of such a leakage, and to the explanation presented here.

A lumbar puncture that I was attempting was interrupted by the nervousness and impending syncope of the patient. I was obliged quickly to withdraw the needle, which I believed from my sense of touch to have already pierced the membranes, without col-

One may be startled by the fact, but can soon convince oneself by a visit to the operating or postmortem room with some needles, that a puncture in this rigid dural membrane usually persists as a clean edged, round hole. The spinal fluid is always under some pressure in its sac, so what is to prevent a continuous leakage into the epidural space in the spinal canal, following the extraction of the needle?

From a study of this point, I have become convinced that closure of the puncture hole usually takes place in the following way: The arachnoid tissue, as it drops from the point of the departing needle, is swept snugly against the dura mater, by the pressure of fluid within. In this way the dural hole is blocked lecting or even seeing any fluid. Nevertheless this patient developed a typical lumbar puncture headache which lasted with severity for eight days.

At the time, of course, this was extremely puzzling. Now it is obvious that an apparently dry tap, if the membranes are pierced, may as truly become one of continuous epidural leakage as any other puncture, and it is continuous epidural leakage of cerebrospinal fluid through a patent puncture hole that is the primary and essential causative factor of lumbar puncture headache. Let us see how this factor operates.

The cerebrospinal fluid contained in a closed sac forms a pad for the brain and spinal cord. At the base of the brain this pad becomes a veritable cushion or 
water-bed. When the fluid leaks away through a hole in the lower end of this sac, the base of the brain loses its supporting fluid cushion. We may expect this support to remain absent until the puncture hole heals and the fluid again fills and distends the sac sufficiently to reestablish this water-bed. The loss of this supporting basal cushion may be regarded as the secondary causative factor in the production of the headache. How does this produce pain?

A headache, which, let us recall, comes on when a patient sits up, and disappears when he lies down, must obviously be mechanically produced. Pressure of the meninges by the brain weight, against the irregular bony surface of the base of the skull, which would ensue when the patient sits up, in the absence of the basal fluid cushion, might be considered to be sufficient cause for headache. However, another explanation appeals to me as more aptly applying to the situation.

A mechanical accident following spinal puncture has caused sudden death in certain brain tumor and other intracranial conditions accompanied by increased intracranial pressure. The withdrawal of fluid deprived the base of the brain of support, and allowed such forcible descent of the pons on the clivus of the occipital bone that prolapse of the medulla through the foramen magnum occurred. In the average normal case if the supporting fluid cushion is completely lost by continuous leakage through a patent puncture, hole, we may expect the pressure of the brain weight transmitted through the pons to the clivus when the patient sits up to be considerable.

The basilar plexus on the clivus of the occipital bone is formed by an extensive anastomosis of flat venous plexuses. It is connected on either side with the cavernous and inferior petrosal sinuses, and with the neighboring blood

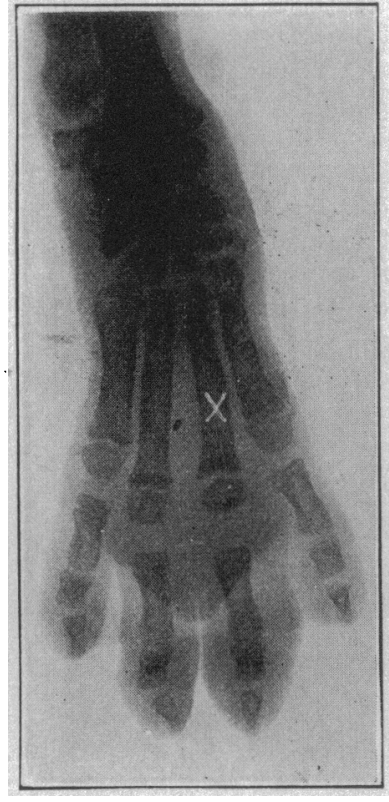

Fig. 1.-Right second metatarsal transposed in position formerly occupied by the left, immediately following operation. channels (Sobotta-McMurrich). The other venous channels that drain the cranial cavity at the base are rigid inelastic tubes, and they are thus safe from closure by pressure. The basilar veins differ in this matter of security from closure. They depend on the cushion of cerebrospinal fluid to keep off the pressure of the pons, which is directly above.

When the patient sits up, and the cushion of fluid is absent, the weight of a good part of the brain is suddenly imparted through the pons to this communicating plexus. The blood about to leave the skull is impeded, and forced to turn back and travel by other crowded pathways. The resulting congestion causes a sudden rise of venous pressure.

The sudden onset of severe headache when the patient sits up can now be understood as due to the sudden heightened intracranial pressure due to the rise of pressure in the cerebral veins; its entire relief, when the patient lies down, as due to the fall of pressure when the weight is removed from the veins

on the clivus. In the course of a week the puncture hole heals, the fluid is rapidly made in sufficient quantity to fill and distend the entire sac, and the integrity of the brain cushion or water-bed is reestablished. The headache, which was a purely mechanical affair dependent on the loss of that cushion, is gone.

20 West Fiftieth. Street.

\section{FURTHER OBSERVATION ON A TRANS- POSED METATARSAL BONE}

$$
\begin{aligned}
& \text { W. L. BROWN, M.D. } \\
& \text { C. P. BROWN, M.D. } \\
& \text { EL PASO, TEXAS }
\end{aligned}
$$

In 1916, we reported ${ }^{1}$ an experiment (Experiment 1 , Dog 7, New Series) in which the second metatarsal bone of a $\operatorname{dog} 10$ weeks old was transposed into the bed left by the removal of the second metatarsal of

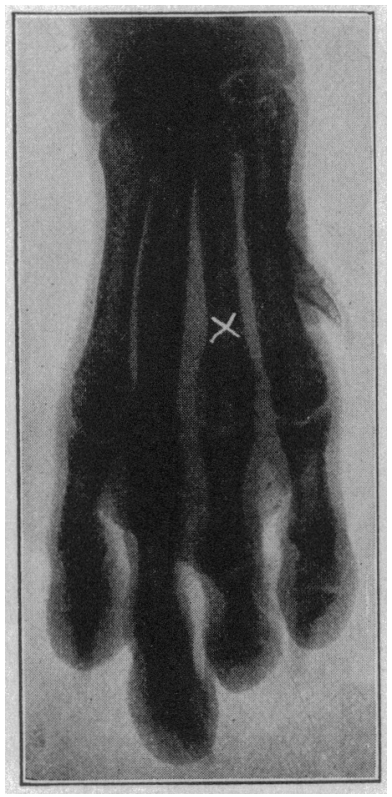

Fig. 2.-Threc months and twenty-six days after transposition: Bone has not only survived and performed its function, but has actually grown as have the other bones

the opposite forefoot. As we stated, three months and twenty-six days after the transposition, the bone had not only survived but had actually grown in length and circumference.

A roentgenogram taken recently reveals that the growth of the transplanted bone has not kept pace with the other bones of the foot either in length or circumference, though it has lived and performed its function for two years.

Conclusions have often been drawn from experiments of short duration, when a more prolonged observation would show that there was a handicap because of the transposition that could not be completely overcome. If this experiment had been done on a dog that remained small, or one already fully grown, the point brought out above would have been missed.

1. Brown, W. L., and Brown, C. P.: Experimentally Transplanted and Transposed Whole Metatarsal Bones, Tile Journal A. M. A. Oct. 21, 1916, p. 1200. 